\title{
Comparación de Dos Métodos Radiológicos para Evaluación de Densidad Ósea en Mujeres Posmenopáusica
}

\author{
Comparison of Two Radiological Methods for Evaluation \\ of Bone Density in Postmenopausal Women
}

Angela Jordão Camargo*; Emiko Saito Arita**; Magda Carolina
Cortéz de Fernández $^{* * *}$ \& Plauto Christopher Aranha Watanabe ${ }^{* * * *}$

CAMARGO, A. J.; ARITA, E. S.; DE FERNÁNDEZ, M. C. C. \& WATANABE, P. C. A. Comparación de dos métodos radiológicos para evaluación de densidad ósea en mujeres posmenopáusicas. Int. J. Morphol., 33(2):732-736, 2015.

RESUMEN: Debido a la importancia del diagnóstico precoz de osteoporosis, fue objeto de este estudio realizar la comparación de dos métodos radiológicos convencionales para la evaluación de la densidad ósea en mujeres posmenopáusicas. Fueron seleccionadas imágenes de radiografía panorámica y de radiografía carpal, correspondiente a 68 pacientes de sexo femenino, con edades comprendidas entre los 49 y 80 años de edad, realizadas en la Clínica de Radiología Odontológica, para evaluación previa a la colocación de implantes. Para la interpretación de la densidad ósea en la mandíbula a través de las radiografías panorámicas fue utilizado el método del espesor cortical mandibular (PMI). Para la evaluación de las imágenes radiológicas obtenidas por la radiografía carpal, fue aplicado el método de la radiogrametría. Se concluye que existe correlación entre la presencia de una baja densidad ósea a nivel mandibular observada en la radiografía panorámica en las pacientes de la muestra, con la baja densidad ósea presentada en la radiografía carpal.

PALABRAS CLAVE: Radiogrametría; Radiografía carpal; Espesura cortical mandibular; Radiografía panorámicas; Densidad ósea.

\section{INTRODUCCIÓN}

La osteoporosis fue definida como "una enfermedad esquelética sistémica", caracterizada por baja masa ósea y deterioro en la micro-arquitectura del tejido óseo, que origina fragilidad ósea, la cual, aumenta el riesgo de fractura (National Institute of Arthritis and Musculoskeletal and Skin Diseases et al., 1991). Actualmente la osteoporosis es definida como "una enfermedad del esqueleto caracterizada por un compromiso en la resistencia del hueso, que predispone a un incremento en el riesgo de fractura" (WHO, 2004).

La Osteoporosis pertenece al grupo de "enfermedades crónicas no transmisibles del adulto", donde además figuran las enfermedades cardiovasculares, las tumorales malignas, y las respiratorias, ocupa un lugar preponderante como causa de muerte en poblaciones que están en transición demográfica, debido al incremento de la expectativa de vida.
La identificación de la baja densidad mineral ósea (DMO) en cualquier sitio del esqueleto a través de la densitometría, es una estrategia común para predecir el futuro riesgo de fractura osteoporótica. Los estudios recientes indican que los marcadores bioquímicos de recambio óseo por si solo o en combinaciones con la DMO, pueden también ser útiles para predecir la futura pérdida de hueso y riesgo de fractura en mujeres de edad avanzada. Sin embargo, no es práctico referirse a todas las mujeres postmenopáusicas para una o ambas de estas medidas cuando uno considera la falta de relación costo-beneficio, además del limitado número de instalaciones y personal capacitado (Taguchi et al., 20003).

Las medidas obtenidas de la cortical mandibular a través de las radiografías dentales panorámicas se asociaron con un aumento del riesgo de fractura osteoporótica, sin

\footnotetext{
* Especialista en radiología dental; Maestría en Ciencias - Área: Diagnóstico Oral, São Paulo, Brasil.

** Especialista en radiología dental, Maestría y Doctorado en Ciencias - Área: Radiología; Profesora de la Facultad de Odontología, Universidad de São Paulo, São Paulo, Brasil.

*** Especialista en radiología dental, São Paulo, Brasil.

***** Especialista en radiología dental, Maestría y Doctorado en Ciencias - Área: Radiología; Profesor de la Facultad de Odontología de Ribeirão Preto, Universidad de São Paulo, Ribeirão Preto, Brasil.
} 
embargo, poco se sabe acerca de las asociaciones de forma $\mathrm{y}$ ancho de cortical mandibular con los marcadores de recambio óseo (Taguchi et al.).

\section{MATERIAL Y METODO}

Las imágenes se obtuvieron de radiografías panorámicas y de radiografías cárpales, correspondientes a sesenta y ocho (68) pacientes mujeres, en edades entre 49 y 80 años. Estas pacientes fueron referidas a la Clínica de Radiología Odontológica, para la correspondiente evaluación previa a la colocación de implantes. Todas estas líneas y medidas fueron diseñadas a través del Software RADIOIMP DE RADIO MEMORY LTDA versión 2.0.

Radiografía Panorámica y Medidas de Índice Cortical Mandibular (IC Mandibular). Las radiografías panorámicas se realizaron en un equipo ORTHRALIX 9200, marca GENDEX, con un patrón aproximado de funcionamiento de: $74 \mathrm{KV}, 10 \mathrm{Ma}$ y $12 \mathrm{~s}$. Las imágenes fueron tomadas con un Chasis KODAK LANEX, Medium de 15 x $30 \mathrm{~cm}$ y con Filmes FWI HR-V30 Súper de la misma medida.

Las medidas fueron obtenidas de manera bilateral en las imágenes panorámicas tomando como referencia el foramen mentual según la técnica de Wical \& Swoope (1974), que consiste en: Diseño del foramen mental, trazado de línea paralela al cuerpo mandibular, trazado de línea perpendicular a la paralela anterior que se proyecta a partir del margen inferior del foramen mental hasta el margen mandibular y trazado de línea paralela a la perpendicular anterior que determine en milímetros $(\mathrm{mm})$ la densidad de la cortical (Fig. 1).
Radiografía Cárpales y Medidas del Índice Cortical Metacarpo (IC Metacarpo). Fueron obtenidas de la mano de menos dominancia del paciente con el mismo equipo Ortomopantografo con que se realizaron las panorámicas, en Chasis Kodak de 20 x $25 \mathrm{~cm}$ y Filmes Fuji HR V30 de la misma medida.

Las medidas fueron obtenidas del índice cortical del $2^{\circ}, 3^{\circ}$ y $4^{\circ}$ metacarpo de la siguiente manera: diseño una línea longitudinal que divida el metacarpo en dos partes iguales a lo largo de su eje máximo; se trazó una línea paralela a la base del hueso que lo corte en su línea mediana, correspondiente al ancho del hueso en estudio (D), según técnica descrita por Dequeker (1976); el mismo nivel de la línea anterior se midió la parte interna o medular (d).

Se realizó la sustracción simple (D) del ancho mesiodistal al ancho medular (d) y obtenemos el IC Metacarpo del $2^{\circ}$, 3er y $4^{\circ}$ metacarpo respectivamente para cada paciente en estudio (Fig. 2).

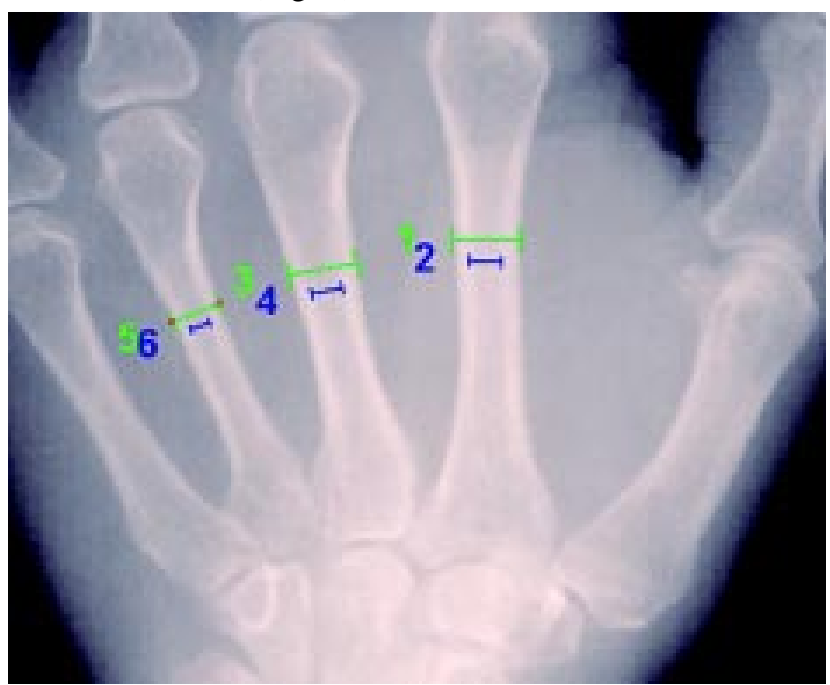

Fig. 2. Sustracción simple del ancho mesio-distal al ancho medular.

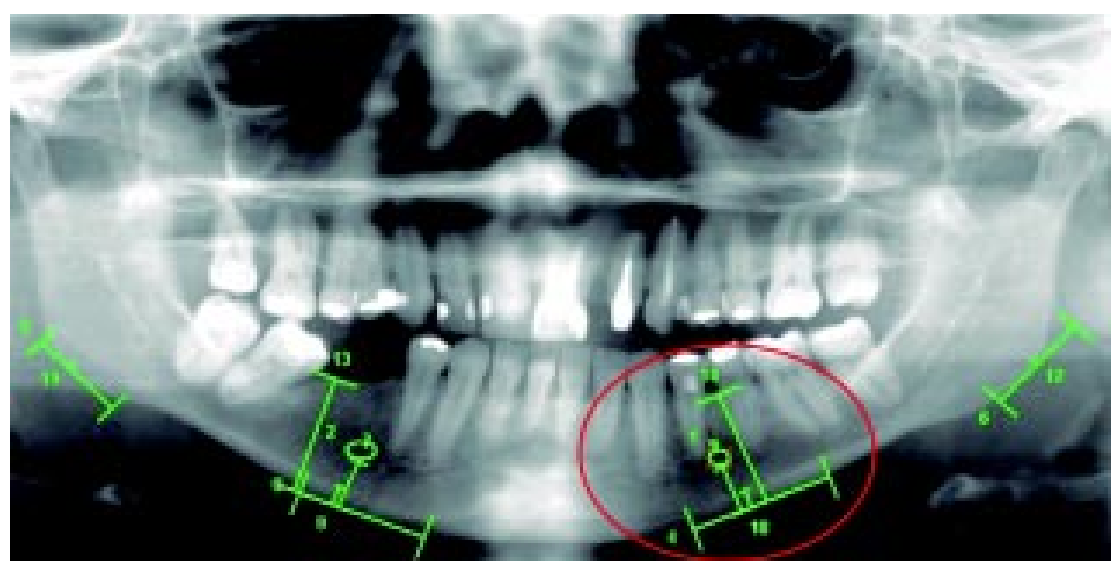

Fig. 1. Medidas de Índice Cortical Mandibular realizado bilateralmente cerca del foramen mental.
Posterior a la exposición, las películas radiográficas se procesaron con equipo automático marca XR24PRO DURR -DENTAL, con un tiempo aproximado de ciclo de $4 \mathrm{~s}$ a una temperatura de $28^{\circ} \mathrm{C}$ aproximadamente.

Posterior a la obtención de las imágenes radiográficas, estas se digitalizaron con un Scanner HP SCANJET 4890, en imágenes tiff, con una resolución de 300 dpi, en escala de grises y salvadas en un Pendrive de la marca KINGSTON de 8GB de capacidad. 
Análisis Estadístico. Los datos para la presente investigación fueron procesados estadísticamente con el software SPSS ver 120 para Windows. La información se presentó en cuadros de distribución de frecuencia y de asociación. En los mismos se muestran frecuencias absolutas y porcentajes y el coeficiente de correlación de Pearson, utilizado para establecer la fuerza de la relación entre los dos tipos de densidad ósea (IC Mandibular - IC Metacarpal). También se dan resultado de las pruebas de significación estadística realizadas a los coeficientes de correlación. También se calculó el promedio y la desviación estándar para la edad de la población investigada. El criterio de significación utilizado en todos los análisis es menor al 5\% de error de la muestra, una probabilidad menor de 0,05 .

\section{RESULTADOS}

La edad promedio de los sujetos en estudio fue de 58,9 años y la desviación estándar de 8,5 años, con una edad mínima de 49 años y una máxima de 80 años.

El 55,9\% del grupo tiene un IC Mandibular Derecho menor ó igual a $3 \mathrm{~mm}$, mientras que el $44,1 \%$ presentó una densidad cortical mandibular superior a los $3 \mathrm{~mm}$ (Tabla I).

$\mathrm{Al}$ relacionarlo con la edad, se obtuvo que la medida menor o igual a $3 \mathrm{~mm}$ fue predominante en los grupos de 60 a 70 años o más, con porcentajes respectivos de $73,3 \%$ y $90,9 \%$; mientras que el grupo de pacientes con más de 3 $\mathrm{mm}$, con una representación porcentual del 59,5\% es decir tenían una buena condición de densidad ósea según el estudio. Se determinó una asociación significativa entre la edad según la clasificación adoptada y la medición del ICMD en según categorías, de acuerdo al valor del chi cuadrado que dio una cifra de 11,370 con dos grados de libertad y un error menor al 3 por mil $(\mathrm{P}<0,003)$.

En cuanto a la correlación de los IC Mandibular e IC Metacarpo en la evaluación de la densidad ósea de mujeres post-menopáusica, se relacionar el IC Mandibular Derecho con el IC Metacarpo se obtuvo que en las pacientes de mayor riesgo osteoporótico; en este caso con un ICM menor o igual a $3 \mathrm{~mm}$, el estudio arrojo resultados de correlación positiva significativa estadísticamente con un margen de error de 0,001 , menor al uno por mil (Tabla II). Del lado izquierdo la relación IC Mandibular con IC Metacarpo no tuvo la misma significancia estadística, arrojando valores de 0,$315 ; 0,325$ y 0,387 respectivamente para el Segundo, Tercero y Cuarto metacarpo; pero cercana a la positividad.

\section{DISCUSIÓN}

En este estudio se tomó una muestra de 68 mujeres posmenopáusicas para determinar la densidad ósea mandibular distal al foramen mental, y se obtuvo como resultado que para una población de una edad promedio de 58,9 años, se determinó que el 55,9\% de la población

Tabla I. Distribución de la población investigada según edad y clasificación del índice cortical mandibular derecho.

\begin{tabular}{lccc}
\hline \multirow{2}{*}{ Edad (años) } & $\mathbf{3} \mathbf{~ m m}$ & $>\mathbf{3} \mathbf{~ m m}$ & \multirow{2}{*}{ Total $(\%)$} \\
\cline { 2 - 3 } & Frecuencia (\%)* & Frecuencia (\%)* & \\
\hline $50-59$ & $17(40,5)$ & $25(59,5)$ & $42(61,7)$ \\
60-69 & $11(76,3)$ & $4(26,7)$ & $15(22,1)$ \\
70 o más & $10(90,9)$ & $1(9,1)$ & $11(16,2)$ \\
Total & $38(55,9)$ & $30(44,1)$ & $68(100,0)$ \\
\hline
\end{tabular}

* Porcentaje en base a sub totales horizontales $(\mathrm{CHI} 2=11,370 ; \mathrm{g} .1 .=2 ; \mathrm{p}<0,003)$.

Tabla II. Coeficientes de correlación de pearson (r) del índice cortical mandibular derecho según espesor con los índices corticales de los metacarpales segundo, tercero y cuarto de la población investigada.

\begin{tabular}{lccc}
\hline $\begin{array}{l}\text { Indice Cortical } \\
\text { Mandibular derecho }\end{array}$ & \multicolumn{3}{c}{ Indice Cortical Metacarpo $(\mathbf{m m})$} \\
\cline { 2 - 4 } & Segundo & Tercero & Cuarto \\
\hline \multirow{2}{*}{$\leq 3 \mathrm{~mm}(\mathrm{n}=38)$} & 0,523 & 0,534 & 0,576 \\
& $(\mathrm{P}<0,001)$ & $(\mathrm{P}<0,001)$ & $(\mathrm{P}<0,001)$ \\
& 0,467 & 0,303 & 0,535 \\
& $(\mathrm{P}<0,009)$ & $(\mathrm{P}<0,103)$ & $(\mathrm{P}<0,002)$ \\
\hline
\end{tabular}


tenía un ICM menor ó igual a $3 \mathrm{~mm}$ del lado derecho, siendo predominante en el grupo de mujeres mayores de 60 años. Mientras que del lado izquierdo el ICM se estableció en un $52,9 \%$ en pacientes con un espesor cortical mandibular menor igual a $3 \mathrm{~mm}$, igualmente predominante en el grupo de mujeres mayores de 60 años. En relación a esto Taguchi et al. (2006) obtuvo como resultados que el $90 \%$ de la población de mujeres post-menopáusicas menores de 65 años tenían una DMO menor de $3 \mathrm{~mm}$ y el $60 \%$ de la misma población tenían osteoporosis; de allí que no diferencia significativa con la DMO de osteoporosis en un grupo intermedio de riesgo con (3,2 $\mathrm{mm}$ a 4,2 $\mathrm{mm}$ ) y el grupo de prevalencia. Ellos sugieren que las mujeres de alto riesgo, definidas con una cortical de $(<3 \mathrm{~mm})$, pueden ser identificadas (Taguchi et al., 2006).

Devlin \& Horner (2002) reportaron que el diagnóstico de una cortical de $3 \mathrm{~mm}$ (o menos) era la sugestión más apropiada para tomar como referencia para la densitometría en 74 mujeres caucásicas.

Resultados obtenidos sugieren que el $73 \%$ de las mujeres con baja densidad ósea pueden ser identificadas por un grupo de odontólogos generales, quienes realizan la lectura en 1 hora; de allí que la obtención del diagnóstico no debe estar influenciada por los años de práctica general de odontología (Taguchi et al., 2006).
Al correlacionar la densidad ósea (IC Metacarpo del segundo, tercero y cuarto metacarpianos de la mano menos dominante en esta investigación; con la densidad ósea encontrada en la cortical mandibular (bilateral) de la misma población se encontró que solo hay una relación significativa para las mujeres con ICM menor ó igual a $3 \mathrm{~mm}(\mathrm{P}<0,05)$ con el índice metacarpo del segundo y cuarto metacarpiano. Del lado izquierdo para las mujeres con ICM menor o igual a $3 \mathrm{~mm}$, se estableció que no hubo en ningún caso relación de orden significativa, ya que los coeficientes estuvieron todos con un error superior al $5 \%$.

La cortical mandibular puede ser evaluada para correlacionarla con la disminución de la densidad mineral ósea del esqueleto, controversialmente se dice que constituye una delgada capa, eso afecta la sensibilidad y especificidad en el diagnóstico. Estos han recomendado que la cortical menor o igual a $4,5 \mathrm{~mm}$. Como indicador de un alto riesgo de osteoporosis. Estos 4,5 mm determinan una alta sensibilidad en la prueba, pudiendo producir un alto número de falsos positivos innecesarios para confirmar densidad mineral ósea examinada (Devlin et al., 2007).

De acuerdo con los resultados de este estudio se puede concluir que existe correlación entre la presencia de una baja densidad ósea a nivel mandibular observada en la radiografía panorámica en las pacientes de la muestra, con la baja densidad ósea presentada en la radiografía carpal.

CAMARgo, A. J.; ARITA, E. S.; DE FERNÁNDEZ, M. C. C. \& WATANABE, P. C. A. Comparison of two radiological methods for evaluation of bone density in postmenopausal women. Int. J. Morphol., 33(2):732-736, 2015.

SUMMARY: Due the importance of diagnosis precocious of osteoporosis, this study carried out the comparison of two conventional radiological methods for the evaluation of bone density in postmenopausal women. X-ray images of panoramic and carpal were selected, corresponding to 68 women between 49 and 80 years old, obtained at the Clinic of Dental Radiology, for holding dental implant. The interpretation of bone density in jaw on panoramic radiography was the method to determine mandibular cortical thickness. The carpal radiography was evaluated for the radiogrammetry method. It was concluded that there was a correlation between low bone density observed in the mandibular panoramic radiography with low bone density on carpal radiography. density.

KEY WORDS: Radiogrammetry; Carpal radiography; Mandibular cortical thickness; Panoramic radiography; Bone

\section{REFERENCIAS BIBLIOGRÁFICAS}

Dequeker, J. Quantitative radiology: radiogrammetry of cortical bone. Br. J. Radiol., 49(587):912-20, 1976.

Devlin, H. \& Horner, K. Mandibular radiomorphometric indices in the diagnosis of reduced skeletal bone mineral density. Osteoporos. Int., 13(5):373-8, 2002.

Devlin, H.; Karayianni, K.; Mitsea, A.; Jacobs, R.; Lindh, C.; van der Stelt, P.; Marjanovic, E.; Adams, J.; Pavitt, S. \& Horner,
K. Diagnosing osteoporosis by using dental panoramic radiographs: the OSTEODENT project. Oral Surg. Oral Med. Oral Pathol. Oral Radiol. Endod., 104(6):821-8, 2007.

National Institute of Arthritis and Musculoskeletal and Skin Diseases; European Foundation for Osteoporosis and Bone Disease \& American National Osteoporosis Foundation. Consensus development conference: prophylaxis and treatment of osteoporosis. Osteoporosis Int., 1(2):114-17, 1991. 
CAMARgo, A. J.; ARITA, E. S.; DE FERNÁNDEZ, M. C. C. \& WATANABE, P. C. A. Comparación de dos métodos radiológicos para evaluación de densidad ósea en mujeres posmenopáusicas. Int. J. Morphol., 33(2):732-736, 2015

Taguchi, A.; Sanada, M.; Krall, E.; Nakamoto, T.; Ohtsuka, M.; Suei, Y.; Tanimoto, K.; Kodama, I.; Tsuda, M. \& Ohama, K. Relationship between dental panoramic radiographic findings and biochemical markers of bone turnover. J. Bone Miner. Res., 18(9):1689-94, 2003.

Taguchi, A.; Tsuda, M.; Ohtsuka, M.; Kodama, I.; Sanada, M.; Nakamoto, T.; Inagaki, K.; Noguchi, T.; Kudo, Y.; Suei, Y.; Tanimoto, K. \& Bollen, A. M. Use of dental panoramic radiographs in identifying younger postmenopausal women with osteoporosis. Osteoporos. Int., 17(3):387-94, 2006.

Wical, K. E. \& Swoope, C. C. Studies of residual ridge resorption. I. Use of panoramic radiographs for evaluation and classification of mandibular resorption. J. Prosthet. Dent., 32(1):7-12, 1974.

World Health Organization (WHO). WHO Scientific Group on the Assessment of Osteoporosis at Primary Health Care Level. Brussels, Summary Meeting Report, 2004. Disponible en: http:/ /www.who.int/chp/topics/Osteoporosis.pdf.

\author{
Dirección para Correspondencia: \\ Angela Jordão Camargo \\ Departamento de Estomatología \\ Salud Pública y Odontología Forense \\ Facultad de Odontología de Ribeirão Preto \\ Universidad de São Paulo \\ Ave. Café s/n, 14040-904 \\ Ribeirão Preto, SP \\ BRASIL
}

Email: angela.camargo@gmail.com

Recibido : 07-11-2014

Aceptado: 01-04-2015 